\title{
1. Access: Digital media can enlarge access to information about heritage destinations
}

\author{
Lorenzo Cantoni and Emanuele Mele
}

\subsection{INTRODUCTION}

In this chapter, a major contribution of information and communication technologies (ICTs) is presented and discussed: the possibility of providing access to unlimited information (and services) about heritage and tourism. The presentation takes place in three sections. First, the opportunities offered by digital media are discussed, with reference to two information-intensive fields: tourism and culture. Second, the issue of information quality is addressed: the problem of so-called 'information overload', that is, of offering a huge amount of information, quite frequently of poor or mediocre quality. This issue can be tackled both from the supply side, by ensuring high/top-quality content, as well as from the demand side, by being able to select quality information out of a plethora of offers. Third, due to the borderless availability of digital information, its adequateness for different publics, with (very) different linguistic and cultural backgrounds, is discussed.

\subsection{THE ELECTRONIC TEXT AND ITS AFFORDANCES}

Digital media (Cantoni and Danowski, 2015) do offer unprecedented opportunities to share and access information: information and communication technologies (ICTs), and especially the internet, have made information available well beyond what was available through previous media. The affordances of digital media are presented here, discussing their impact when it comes to the 'Access' layer. This section tackles the characteristics of the electronic text as proposed by Cantoni and Tardini (2007, pp. 54-57).

\subsubsection{Duplicable}

Digital media can be easily and endlessly duplicated. When one accesses a webpage, what is accessed is not an 'original' - as it would be when receiving a book from a library's loan service - but a copy, produced on the fly by the webserver. In the digital world, there is no longer a distinction between 'original' and 'copy': everything can be technically copied without any loss of quality. This characteristic reminds us of the debate started by Walter Bejamin (1936 [1968]) and earlier - in a literary form - by the Italian Nobel laureate Luigi Pirandello in his 1925 Quaderni di Serafino Gubbio operatore (Pirandello, 1994; first published in 1916 as Si gira ...): what distinguishes the original from its copies? From an accessibility viewpoint, if being able to copy without any limit is a clear advantage, we need to stress that the copied objects are digital. When it comes to physical/analogical cultural artefacts, the distinction between the original and its copies is key. The same can be said about tourism as 
an experience: visiting a place and staying there cannot be fully substituted by any possible digital 'copy' or representation.

\subsubsection{Accessible}

However, digital technologies can provide rich opportunities to access (some) information without (or before) visiting a destination or an attraction. Digital media can be accessed from everywhere, provided the proper digital infrastructure is available, and there is no censorship or filtering.

Accessing information can be either a way for potential tourists to get inspiration, before eventually deciding to visit a place, or a way to better plan the visit. On other occasions, travellers may access content on the web for purposes other than inspiration or planning.

Within the tourism context, digital technologies can provide a surrogate experience for those who cannot visit a place for subjective or objective reasons; for example, due to economic, legal, logistic or disability issues. The Covid-19 pandemic has made this aspect even clearer. On the one hand, people have not been able to move because of health and safety measures in the form of lockdowns and travel limitations. On the other hand, digital technologies have emerged as the major way, and perhaps the only possible way, to see and virtually visit travel places or enjoy cultural events.

Mere technical accessibility is not enough to reach the intended publics. Never in the past has so much information been available to interested people. While the issue of its quality will be tackled in the next section, it is important to mention here the attribute of 'findability': publishers need to invest notable resources not only to produce quality content, but also to ensure that it is found by (or, conversely, it reaches) its interested public. In an information overload context, abundance of information might also mean its loss, in which case the 'noise' could become the only message conveyed to the receiver.

\subsubsection{Flexible}

Cultural tourism refers to a plethora of facets and dimensions (e.g., Timothy, 2011), subject to interpretation changes and research integrations. This characteristic is matched by the flexibility of the electronic text: every piece of content can be constantly improved, revised, enriched and reinterpreted. Multiple voices presenting the same place, from different perspectives and with different interpretations, can be orchestrated in the digital realm to enable a constant improvement in the touristic communication.

In particular, the case of the so-called 'user-generated content' (UGC) must be mentioned here. Before the advent of Web 2.0 (Cantoni and Tardini, 2010), most of the touristic-related content was published by specialized agencies. Nowadays, individual travellers and interested people can publish travel reviews and other content online (De Ascaniis and Gretzel, 2013), co-creating the image and information market relating to destinations and attractions (Marchiori and Cantoni, 2011).

\subsubsection{Interactive}

A fundamental characteristic of digital media, connected with its flexibility, is the possibility for the user to interact with the content/application, thus designing usage paths that could not 
be anticipated at first (Cantoni and Paolini, 2001). The usage of the electronic text is always a dialogue or interaction between the content and the reader, as suggested by Umberto Eco in his Lector in fabula (Eco, 1979).

Moreover, this interactivity enables a seamless move from accessing information to making transactions: a digital tourism/cultural platform can always hybridize the support for knowledge building and for doing business.

\subsubsection{Customizable}

The flexibility and interactivity outlined above provide extensive room for customization. More precisely, in cultural tourism there is the possibility to design dedicated offers and messages to different persons, depending on their interests, which can be stated explicitly or inferred by the system itself from demographic or behavioural data. Recommender systems (Ricci et al., 2011) might provide more relevant contents, which in turn might be better understood and received by the addressees. In the context of the so-called 'attention economy' (Davenport and Beck, 2002), serving personalized content might mean winning more attention and, eventually, more interested visitors.

\subsubsection{Multimedia}

So far, we have referred to the electronic text without further specifications. At this point, it is important to stress that this 'text' can mix all semiotic codes: textual, as well as images, videos, audio, and so on.

While the senses of touch, smell and taste have still very limited presence in the digital world, sight and hearing are extensively covered. Consequently, beside fully supporting the idea of tourism as 'seeing' a place (Urry and Larsen, 2011), digital media are more and more (re)presenting soundscapes, anticipating in a more vivid way what it could mean to stay in a place.

Virtual/augmented reality (VR/AR) and mixed realities are emerging in the field of cultural tourism, providing endless opportunities to complement what is accessed during a visit (hence, in the 'Better' layer of ABCDE (Cantoni, 2018)), as well as presenting computer-generated worlds, which offer a substitute experience of something that cannot be directly accessed, such as the ancient world, the deep sea and destroyed historical sites (Marchiori and Cantoni, 2020; Videva et al., 2019).

\subsubsection{Persistent (Or Not?)}

While digital media can be considered 'fluid' or 'liquid' objects, as our current societies (Bauman and Haugaard, 2008), they are also persistent: most events in the digital realm are recorded and leave a trace. This dynamic opens the possibility to analyse, for instance, actual instances of user-content interaction via digital analytics. Data extrapolated from user navigation can be interpreted and used by online communication managers to improve content in terms of both depth (richness) and relevance.

This persistency coexists with digital technology's obsolescence and its short life cycle, if compared with previous media. Hardware, software and digitizing standards change at a very fast pace, requiring that digital assets are constantly updated and refreshed, to keep 
them 'alive' and still accessible. While in many cases this aspect is neglected in new media discourses (sometimes leading to incorrect investment forecasts), it is important to mention it here, in a context in which culture and heritage are discussed. While museums and archives are used to conserving culturally relevant objects for centuries, ensuring the correct physical environment and necessary restoration, digital objects need constant monitoring and updating to the latest standards in order not to lose their communication power.

Following this discussion on the intrinsic characteristics of digital media, which are particularly relevant for the 'Access' layer, we now need to focus on quality criteria.

\subsection{INFORMATION QUALITY}

Providing information access for heritage/cultural tourism requires a strong focus on quality (Eppler and Witting, 2000). In general, quality can be described 'as conformance to specifications ... [or] as meeting or exceeding customer expectations' (Kahn and Strong, 1998, p. 102). Information quality, then, 'can be defined as the characteristic of information to meet or exceed customer expectations; and quality information is information that meets specifications or requirements' (Cantoni and Tardini, 2007, p. 102). The two approaches are closely linked: specifications are defined in order to ensure fit with users' requirements.

According to Alexander and Tate (1999; see also Tate, 2019), who adapted criteria developed for past media to the digital context, five main criteria should be considered when evaluating content quality: accuracy, authority, coverage, currency and objectivity (see also Cantoni and Tardini, 2007, and, for a larger debate, Eppler and Witting, 2000). In this section, the issue will be approached at the three different levels indicated by Morris (see Allwood, 1981) as being relevant when studying human communication: semantic, syntactic and pragmatic.

\subsubsection{Semantic Level}

At the semantic level, an adequate representation of the expressed (semantic) universes is to be ensured. As was pointed out in medieval philosophy, a certain adequateness between the message and reality should be attained. While at the factual level such accuracy might be straightforward - for example, in referring to Istanbul or Cyprus, I need to provide the correct geographical framing - at the interpretation level, semantic accuracy is more a target than something that can be attained once and for all: for example, is it Istanbul or Constantinople?

Cultural objects and issues are to be presented by outlining their complexity and interpretation challenges. This does not mean attaining an (impossible) objectivity, a sort of divine omniscience. Rather, it means providing (some) relevant content and interpretation, while acknowledging limitations and declaring the positioning of the authors themselves. In general, it is of the utmost importance that the user can understand which interests are at stake. If a theatre or a museum recommends a hotel, it should be clear whether this is done because the hotel has paid them, or based on other reasons, such as proximity, web link exchange or quality assessment.

As mentioned above, digital technologies provide extensive opportunities to cover different topics from multiple angles, using multiple media. Such affordances are to be used wisely here, presenting different voices and perspectives on the same objects and issues. Cultural/ 
heritage tourists are particularly demanding at the semantic level, which requires the use of correct and up-to-date information as well as adequate hermeneutical approaches.

Due to their flexibility, digital media allow for constant changes and updates. In some cases, that has led to a suboptimal publishing process: content is made available without careful proofreading and error checking, assuming that further improvements will be always possible. The fact that online publishing has become particularly simple and accessible to laypersons has meant, in some cases, a reduction in terms of quality: first one publishes, then one (hopefully) checks and improves the published content.

A major issue to be considered here is that of information currency. While with previous media it was sufficiently clear when information had been published, clarifying at which point it attained reality, nowadays, due to the flexibility of the electronic text underlined above, readers will assume that what they access online is current, otherwise it would have been updated or removed. Many websites and other publication outlets are poor when it comes to currency: online information that was true for a certain period becomes false and misleading if left unchanged. For example, opening hours may change due to regular events (for example, seasons) or unforeseeable ones (for example, coronavirus); future events announced in a 'News and Events' section all of a sudden can become something that belongs to the past: 'The presentation of the book on Ethiopian Christian Heritage will take place on 25 March 2022 ' enters the present and immediately afterwards the past on 25 March 2022. The need for constant updating stresses the importance of careful management of digital communication. Digital media are neither only nor mainly products: they are processes, requiring major efforts to keep them up to date.

The need for constant updating is particularly high in tourism, where specific elements do change quickly, such as the cost or availability of tickets, events and timetables. Yet, some cultural-related pieces of information might be quite stable: the painter of a fresco, if the attribution is not challenged, is forever the same. This is a relevant challenge for a digital publication related to cultural tourism. In some cases, for instance, it is better to clearly distinguish the two domains, so that different persons curate and update different pieces of information through ad hoc procedures. In this regard, it is possible to take the example of a mobile app about a World Heritage Site. The description and interpretation of the property might be rather stable (even if always open to improvements and integrations), while tourism-related information is likely to change at high speed; for instance, concerning hotels, restaurants, events and transportation. Instead of releasing the app with tourism data, which are likely to make it obsolete in a few months, one might choose to put only links towards the relevant destination management organization, which is fully equipped to ensure constant updating of the tourism offers. Alternatively, different players might ensure the availability of their data/information through dedicated interfaces and syntax, so that everyone looks after their own domain, while the travellers can enjoy a full, constantly updated offer.

This last example stresses a very interesting element in this field: the issue of coverage. Digital media are constantly (re)negotiating their communication territories and boundaries, seeking to define new 'literary genres'. In this regard, posters, magazines or television ads used to be quite stable for decades. The same can be said, for instance, about catalogues and brochures. Digital media have made these literary genres and their boundaries extremely blurry, asking for a constant reinvention of them. For instance, people nowadays can interact with an Instagram image to buy a product or a service; a poster can link directly - through a QR (quick response) code - to a discounted offer. 
The coverage challenge is not only due to new digital affordances; it goes deeper than that. It requires a redefinition of what a publication outlet is all about. A few years ago, a museum catalogue and a hotel brochure were clearly different objects; nowadays hotel websites are integrating information about what to visit and events in their area, while museum apps can provide recommendations of restaurants and hotels. Coverage-related issues are also emerging because digital media do not obey those physical laws that contributed to define previous literary genres. Space and weight had dictated the dimensions of brochures and catalogues, and specific publication channels limited the available semiotic codes; for example, printed media do not allow for audios and videos. Nowadays we need to redefine such genres, having in mind, on the one hand, the new media affordances and the new available publication platforms; on the other hand, the different business goals and how such media can help to reach them. And last but not least, considering emerging and changing usage practices on the side of the addressees.

\subsubsection{Syntactic Level}

While an adequate relationship between information and the world it refers to is addressed at the semantic level, the formal accuracy of the message itself is considered at the syntactic level.

The first form of correctness refers to grammar, which is frequently violated; most probably because of the excessive reliance on the flexibility of digital media, following the idea that every mistake will eventually be fixed. A major issue here is also connected with multilingual content and translation. Indeed, tourism requires that a common language is used to communicate a message, ideally the native language of the client or prospect.

An easier management of multilingualism is an advantage offered by digital media, such as the possibility of providing multiple translations of exhibition labels using QR codes, without having to enlarge the labels themselves. Yet, such opportunities might result in poor-quality translation, if not properly managed from the viewpoint of interface and update as well as in terms of accuracy. Indeed, keeping a multilingual website up to date means multiplying all the publication activities. If automatic translation is used, it might work well on quite simple texts, based on standardized items. Yet, it might become quite misleading - or even lead to amusing outcomes - when dealing with complex cultural content.

An additional source of poor accuracy might result from the digitization of documents previously available only on paper. While in many cases the quality of automatic character recognition might be exceptional, even small errors in the field of cultural tourism might be highly problematic, such as mistakes concerning dates.

Until now, we have focused mainly on the information side of digital media. Yet, it is also important to mention the functional elements and usability issues. These range from providing clear information about a theatrical play, including cultural and more practical elements, to booking and buying a ticket, which can be a very smooth process if enabled by a well-designed booking engine. The same applies to museums, galleries, hotels and theme parks. Content quality cannot be fully disjointed from the quality of the user experience itself: a poorly designed interface might limit the navigation or even impair the possibility of a successful interaction. 


\subsubsection{Pragmatic Level}

The pragmatic approach studies how communication articulates the relationship between the persons involved. In this area we need to tackle first the issue of authority: who is the sender of the communication? In previous media, the identification of senders was usually rather clear; in most cases only a few authors were able to publish, ensuring a certain quality (or at least a selection process). As we have seen, that might also have limited dramatically the richness of available voices and narratives, excluding or even censoring some of them. When it comes to digital media, knowing the author(s) of a message has become more difficult, and at times even impossible. Yet, within the tourism and cultural context, it is very important that readers/users can understand who the senders are, whether and why they should be trusted. This dynamic encompasses the domain of cultural interpretation as well as that of business: has what I see in an augmented reality app been designed by a competent authority in history or archeology, or is it the naïve invention of a bricoleur? Is the information I get about opening hours offered by the business itself, or by an improvised tour operator?

Within this context, user-generated content has opened plenty of new opportunities and challenges at the same time. Through online travel reviews (De Ascaniis and Cantoni, 2018), visitors co-create the available narratives about destinations, attractions and tourism players, while making it very difficult to identify authors' actual identity and their authority when it comes to their statements. This issue has no simple solution. Publishing platforms should implement policies to avoid simulation and fabrication: the content can be considered meaningful as long as the authors can be clearly identified. For instance, if I see a video liked by 100 000 people, I should be quite confident that those who liked it were actual users, with access to the video, and not fake accounts guided by a smart programmer. If a review is published about a hotel or an attraction, the writer should be identifiable in order to provide enough certainty that they were an actual client. The flexibility of digital media is raising more issues than it provides solutions and answers to; however, such answers cannot be found exclusively - or mainly - in technical or policy interventions: above all, they should be looked for in education and in a constant upskilling of users, and the citizenship at large.

If we want to orchestrate all three elements that are the subject of this Handbook - digital media, tourism and heritage - it is important to highlight here that heritage communication has to embrace new forms, which go beyond the pragmatic relations of expert-learner and expertexpert. In tourism, we need to factor in a major component of entertainment. Considering the affordances of digital media, this is usually done through storytelling (Pera, 2017) and gamification (Adukaite et al., 2017). These strategies help promote interest and pleasure, while supporting knowledge sharing and memorability. So long as this is not done at the expense of correctness and does not compromise on scientific quality, it is a major contribution to effective communication.

\subsection{LOCALIZATION}

Localization refers to a set of activities aimed at adapting a product for a specific geographical market. Within the realm of online communication, this set of activities refers to the adaptation of multimedia content (images, videos and text) and layout - whenever possible - to suit the cultural background of a specific domestic or international audience (Mele and Cantoni, 
2018). In other words, localized content arguably becomes more accessible and understandable to people browsing the web from a specific country or region.

Translation can be considered the basis of any localization activity, which transposes the source language to the target language spoken by the receiver. Translation has a clear and direct relation with content accessibility. The website of a company or organization, such as a destination marketing organization (DMO), will gain visibility among search engine results if the textual content has been translated by a person who is also expert in search engine optimization (SEO) (Mele and Cantoni, 2018). Indeed, following a query in the target language (for example, Chinese), the search engine will display the webpages from its database that contain the searched terms (keywords) provided by the user.

For example, if a prospective tourist from China is looking for information online (in Chinese) on the main attractions to visit in Italy, the search engine may show the Italian DMO website among the main results, as it has a version localized for the Chinese market (ENIT - Italian National Tourist Board, 2020). Consequently, the content promoting Italian attractions and cities would become accessible to that market. Within this context, SEO is relevant because the translator will have to provide to the search engine the appropriate keywords (among other elements) to reach a satisfactory positioning of the website among the results displayed to the user after the query.

Translation can be also useful for content published on social media, which represent one of the most important sources of inspiration for tourists (Molinillo et al., 2018). Among the social networks, Instagram is an extremely popular image-based channel, used by several DMOs to keep in contact with visitors and attract new ones (Uşaklı et al., 2017). One way for users to search content on Instagram is by hashtags (\#). Consequently, to ensure that the translated post will appear after a search from a specific market (for example, Italy), DMOs often include ad hoc hashtags in the language of the audience (for example, Italian). This technique also allows visitors to post content on Instagram about their experience at the destination by using a hashtag in a language they know, to make it visible (and accessible) to the DMO itself. This is the case, for example, of the Swiss DMO 'MySwitzerland' (Switzerland Tourism, 2020), which has Instagram accounts in multiple languages, each dedicated to a specific market.

In addition to the technical accessibility of online content, translation is also important as concerns all those elements that are highly dependent on the geographical and cultural context, such as units of measurement (for example, imperial versus metric system), calendars, laws, expressions and metaphors (Cantoni and Tardini, 2007). For example, the British expression 'it's raining cats and dogs' does not have a literal equivalent in Italian (which would sound rather odd); hence, a replacement would be necessary if this market was to be addressed. Therefore, in this case, translation helps the reader to access the meaning behind expressions; that is, it facilitates the understanding of content.

Nevertheless, there are cases in which translation is not enough to make content fully understandable to visitors from a specific market. Indeed, they may lack the necessary cultural background to understand the meaning behind a word or concept. Therefore, it could be required - from the editor's side - to add or remove pieces of content connected to an attraction or destination, thus manipulating information granularity (Mele and Cantoni, 2018). Information granularity expresses the depth and scope of a message, which are a function of the cultural background (and geographical proximity) of the receiver.

For example, the Italian DMO website in the Chinese version (ENIT-Italian National Tourist Board, 2020) summarizes the whole history of the Italian peninsula in a few paragraphs 
(broad scope, less depth), while the French version focuses on specific historical periods and attractions (narrower scope, greater depth). This content choice follows the assumption that details about the history of a culturally and geographically distant place may not be meaningful and appealing for online visitors who have possibly never been to the destination, such as Chinese visitors. Therefore, a broader picture could be more suitable.

These types of localization activities often address the more external, visible layers of a culture. Hofstede et al. (2010) describe these layers as the 'practices' that characterize a society or group of people, consisting of symbols, heroes (historical or mythical) and rituals. Yet, localization can also address more 'hidden' layers, namely the cultural values. These can be defined as people's 'broad tendencies to prefer certain states of affairs over others' (Hofstede et al., 2010, p. 9), influencing their behaviour both offline (e.g., Seongseop and McKercher, 2011) and online (e.g., Goodrich and de Mooij, 2014). According to Hofstede et al. (2010), cultures can be measured at the national level along the so-called 'cultural dimensions', which are virtual constructs that can be used by researchers as well as practitioners to compare national cultures in terms of cultural values.

Scholars have developed six cultural dimensions (Hofstede et al., 2010). For the scope of this overview, only 'Collectivism vs. Individualism' (COL-IND) will be presented - a dimension that has frequently been used in marketing research (Saleem and Larimo, 2017). Following Hofstede et al. (2010), COL-IND describes the extent to which members of a society are bound to in-groups. In more collectivist cultures (for example, Peru), an individual is part of a large in-group (extended family and friends) and identifies herself/himself with it (as 'we'). In contrast, in more individualist cultures (for example, the Netherlands) people are expected to be more self-reliant and independent (as 'I'). From a tourism communication perspective, content emphasizing collectivist cues may depict experiences with family and friends, whereas individualist cues may be represented by feelings of independence and self-fulfillment (Mele et al., 2020).

The localization of cultural values is meant to make content resonate with the cultural background of the audience, arguably making it more meaningful as well as appealing. Within the tourism domain, so far only two empirical studies (one of them limited to a European context) have tested the effects of localizing cultural values on online visitors' perceptions and intentions (Mele et al., 2020; Tigre Moura et al., 2014), and there is a research gap when it comes to the effect of manipulating information granularity. Therefore, future research should focus on localization effects using an experimental design, with participants from culturally as well as geographically distant countries.

Finally, localization could be also explored in terms of its relationship with perceived familiarity (cultural closeness) and novelty. Indeed, excessive localization (over-localization) could lead the sender (for example, the DMO) to promote the destination in a way that resembles the receiver's home. In other words, the effort to make the prospective tourist comfortable by smoothing away cultural differences could lead to a loss of perceived authenticity and downplay the novelty represented by the destination. Therefore, it is argued that a communicative balance should be found between culturally congruent content and features emphasizing the uniqueness (and novelty) of a destination (Mele and Cantoni, 2018). 


\section{REFERENCES}

Adukaite, A., I. van Zyl, Ş. Er and L. Cantoni (2017), 'Teacher perceptions on the use of digital gamified learning in tourism education: the case of South African secondary schools', Computers and Education, 111, 172-190, doi: 10.1016/j.compedu.2017.04.008.

Alexander, Janet E. and Marsha Ann Tate (1999), Web Wisdom: How to Evaluate and Create Information Quality on the Web, Mahwah, NJ: Lawrence Erlbaum Associates.

Allwood, Jens (1981), 'On the distinctions between semantics and pragmatics', in Wolfgang Klein and W. Levelt (eds), Crossing the Boundaries in Linguistics, Synthese Language Library (Texts and Studies in Linguistics and Philosophy), 13, Dordrecht: Springer, pp. 177-189.

Bauman, Z. and M. Haugaard (2008), 'Liquid modernity and power: a dialogue with Zygmunt Bauman', Journal of Power, 1 (2), 111-130, doi: 10.1080/17540290802227536.

Benjamin, Walter (1936 [1968]), 'The work of art in the age of mechanical reproduction', in Hannah Arendt (ed.), Illuminations, New York: Schocken Books (1968), pp. 217-252.

Cantoni, Lorenzo (2018), 'eTourism for heritage: heritage and sustainable tourism. The role and challenge of information and communication technologies', in Silvia De Ascaniis, Maria Gravari-Barbas and Lorenzo Cantoni (eds), Tourism Management at UNESCO World Heritage Sites, Lugano, CH: USI - Università della Svizzera italiana Press, pp. 67-73.

Cantoni, Lorenzo and James A. Danowski (eds) (2015), Communication and Technology, Berlin, Germany and Boston, MA, USA: De Gruyter.

Cantoni, L. and P. Paolini (2001), 'Hypermedia analysis: some insights from semiotics and ancient rhetoric', Studies in Communication Sciences, 1, 33-53, doi: http://dx.doi.org/10.5169/seals-791133.

Cantoni, Lorenzo and Stefano Tardini (2007), Internet, London: Routledge.

Cantoni, Lorenzo and Stefano Tardini (2010), 'The Internet and the Web', in Daniele Albertazzi and Paul Cobley (eds), The Media. An Introduction (3rd edn), New York: Routledge, pp. 220-232.

Davenport, Thomas and John Beck (2002), The Attention Economy: Understanding the New Currency of Business, Cambridge, MA: Harvard Business Press.

De Ascaniis, Silvia and Lorenzo Cantoni (2018), 'Social media analysis from a communication perspective: the case of the argumentative analysis of online travel reviews', in Marianna Sigala and Ulrike Gretzel (eds), Advances in Social Media for Travel, Tourism and Hospitality. New Perspectives, Practice and Cases, New York: Routledge, pp. 262-276.

De Ascaniis, S. and U. Gretzel (2013), 'Communicative functions of online travel review titles: a pragmatic and linguistic investigation of destination and attraction OTR titles', Studies in Communication Sciences, 13 (2), 156-165, doi: 10.1016/j.scoms.2013.11.001.

Eco, Umberto (1979), Lector in fabula, Milan: Bompiani.

ENIT - Italian National Tourist Board (2020), 'History and art', accessed 19 November 2020 at http:// visitaly.com.cn/theme.php.

Eppler, Martin and Doerte Witting (2000), 'Conceptualizing information quality: a review of information quality frameworks from the last ten years', in Barbara D. Klein and Donald F. Rossin (eds), Proceedings of the 2000 Conference on Information Quality, Cambridge, MA: MIT Press, pp. 83-91.

Goodrich, K. and M. de Mooij (2014), 'How "social" are social media? A cross-cultural comparison and offline purchase decision influences', Journal of Marketing Communications, 20 (1-2), 103-116, doi: 10.1080/13527266.2013.797773.

Hofstede, Geert H., Gert Jan Hofstede and Michael Minkov (2010), Cultures and Organizations: software of the Mind (3rd edn), New York: McGraw-Hill.

Kahn, Beverly and Diane M. Strong (1998), 'Product and service performance model for information quality: an update', in Indushobha Chengalur-Smith and Leo Lawrence Pipino (eds), Proceedings of the 1998 Conference of Information Quality, Cambridge, MA: MIT Press, pp. 102-115.

Marchiori, E. and L. Cantoni (2011), 'The online reputation construct: does it matter for the tourism domain? A literature review on destinations' online reputation', Information Technology and Tourism, 13 (3), 139-159, doi: http://dx.doi.org/10.3727/109830512X13283928066715.

Marchiori Elena and Lorenzo Cantoni (2020), 'The relevance of eye-tracking to understand users' practices and content interpretation in tourism-related online navigation', in Mattia Rainoldi and M. Jooss (eds), Eye Tracking in Tourism. Tourism on the Verge, Cham: Springer, pp.71-84. 


\section{Handbook on heritage, sustainable tourism and digital media}

Mele, Emanuele and Lorenzo Cantoni (2018), 'Localization of tourism destinations' websites: theory and practices', in Magdalena Bielenia-Grajewska and María Enriqueta Cortés de los Ríos (eds), Innovative Perspectives on Tourism Discourse, Hershey, PA: IGI Global.

Mele, E., P. Kerkhof and L. Cantoni (2020), 'Cultural localization in online heritage promotion', Journal of Heritage Tourism, 1-17, doi:10.1080/1743873X.2020.1779731.

Molinillo, S., F. Liebana-Cabanillas, R. Anaya-Sanchez and D. Buhalis (2018), 'DMO online platforms: image and intention to visit', Tourism Management, 65, 116-130, doi: 10.1016/j.tourman.2017.09.021.

Pera, Rebecca (2017), 'Empowering the new traveller: storytelling as a co-creative behaviour in tourism', Current Issues in Tourism, 20 (4), 331-338, doi: 10.1080/13683500.2014.982520.

Pirandello, Luigi (1994), Quaderni di Serafino Gubbio operatore, Firenze: Giunti.

Ricci, Francesco, Lior Rokach and Bracha Shapira (eds) (2011), Recommender Systems Handbook, Boston, MA: Springer.

Saleem, Salman and Jorma Larimo (2017), 'Hofstede cultural framework and advertising research: an assessment of the literature', in George Christodoulides, Anastasia Stathopoulou and Martin Eisend (eds), Advances in Advertising Research, Vol. VII, Wiesbaden: Springer, pp. 247-263.

Seongseop Kim, Samuel and B. McKercher (2011), 'The collective effect of national culture and tourist culture on tourist behavior', Journal of Travel and Tourism Marketing, 28 (2), 145-164.

Switzerland Tourism (2020), 'Homepage', accessed 19 November 2020 at https://www.myswitzerland .com/en/.

Tate, Marsha Ann (2019), Web Wisdom, Boca Raton, CA: CRC Press.

Tigre Moura, F., J. Gnoth and K.R. Deans (2014), 'Localizing cultural values on tourism destination websites. The effects on users' willingness to travel and destination image', Journal of Travel Research, 54 (4), 528-542, doi: 10.1177/0047287514522873.

Timothy, Dallen J. (2011), Cultural Heritage and Tourism: An Introduction, Bristol: Channel View Publications.

Urry, John and Jonas Larsen (2011), The Tourist Gaze 3.0, London: SAGE.

Uşaklı, A., B. Koç and S. Sönmez (2017), 'How "social" are destinations? Examining European DMO social media usage', Journal of Destination Marketing and Management, 6 (2), 136-149, doi: 10.1016/j.jdmm.2017.02.001.

Videva, J., E. Marchiori and L. Cantoni (2019), 'Assessing usability and user experience of immersive web VR platforms for tourism destinations', E-review of Tourism Research, 17 (2), https://ertr-ojs -tamu.tdl.org/ertr/index.php/ertr/article/view/509 